\title{
The Protection motivation theory for predict intention of COVID-19 vaccination in Iran: A structural equation modeling approach
}

Alireza Ansari-Moghaddam

Zahedan University of Medical Sciences

Maryam Seraji

Zahedan University of Medical Sciences

Zahra Sharafi

Zahedan University of Medical Sciences

Mahdi Mohammadi

Zahedan University of Medical Sciences

Hassan Okati-Aliabad ( $\nabla$ dr.okati@zaums.ac.ir)

Zahedan University of Medical Sciences

\section{Research Article}

Keywords: COVID-19, vaccination, intention, Structural Equation Modeling, Iran

Posted Date: February 15th, 2021

DOI: https://doi.org/10.21203/rs.3.rs-239756/v1

License: (c) (1) This work is licensed under a Creative Commons Attribution 4.0 International License. Read Full License 


\section{Abstract}

\section{Background}

Many efforts are being made around the world to discover the vaccine against COVID-19. After discovering the vaccine, its acceptance by individuals is a fundamental issue for disease control. This study aimed to examine COVID-19 vaccination intention determinants based on the protection motivation theory (PMT).

\section{Methods}

We conducted a cross-sectional study in the Iranian adult population and surveyed study participants from the first to the 30th of June 2020 with a web-based self-administered questionnaire. We used Structural Equation Modeling (SEM) to investigate the interrelationship between COVID-19 vaccination intention and perceived susceptibility, perceived severity, perceived self-efficacy, and perceived response efficacy.

\section{Results}

SEM showed that perceived severity to COVID-19 $(\beta=.17, p<.001)$, perceived self-efficacy about receiving the COVID-19 vaccine $(\beta=.26, p<.001)$, and the perceived response efficacy of the COVID-19 vaccine $(\beta=.70, p<.001)$ were significant predictors of vaccination intention. PMT accounted for $61.5 \%$ of the variance in intention to COVID-19 vaccination, and response efficacy was the strongest predictor of COVID-19 vaccination intention.

\section{Conclusions}

This study found the PMT constructs are useful in predicting COVID-19 vaccination intention. Programs designed to increase the vaccination rate after discovering the COVID-19 vaccine can include interventions on the severity of the COVID-19, the self-efficacy of individuals receiving the vaccine, and the effectiveness of the vaccine in preventing infection.

\section{Background}

Vaccines are one of the cost-effective measures of prevention (1). Immunization against infectious diseases annually prevents 2-3 million deaths by affecting the immune system (2). The spread of COVID19 as an emerging disease in the world requires immediate action, including the production of vaccines, which can be an effective measure to protect people against this disease (3). Many efforts are being to 
prevent individuals from getting COVID-19 through vaccination (4). After providing the vaccine, the critical issue is its acceptance by the individuals. A survey of American adults found that $69 \%$ of them will accept COVID-19 vaccination (5). A report from the Centers for Disease Control and Prevention found that less than half of American adults vaccinated against the flu in the 2018-2019 season (6).

Evidence shows that the rate of influenza vaccination is low in Asian populations (7), and this rate in Iran is much lower than expected by the World Health Organization (8); however, Iran is one of the countries that announced the highest agreement on the importance of the vaccine (9). The evidence shows that misconceptions are among the main reasons for not getting the flu vaccine (10).

According to a global report in 2017, more than $90 \%$ of countries report that people are hesitant about vaccination (11). Factors affecting COVID-19 vaccination acceptance may be as important as the discovery of the vaccine (12). It is unclear how effective the pandemic status is in accepting the COVID-19 vaccine, and doubts about the vaccine acceptance remain (13). Policymakers can identify factors related to vaccine acceptance to guide effective interventions to increase vaccination acceptance in the population (14). Behavioral change interventions widely use fear appeal to be useful. Fear appeals when messages contain a description of perceived susceptibility, perceived severity, and expressions of response efficacy can positively affect individuals' knowledge, attitude, and performance, especially in onetime behaviors (e.g., Covid-19 vaccination) $(15,16)$.

A recent study examining the effectiveness of the PMT in predicting seasonal influenza vaccination intent has shown that this model is a good predictor (17). To the best of our knowledge, no studies have so far examined the predictors of intention to vaccinate COVID-19 using the PMT. This study aimed to investigate the predictors of COVID-19 vaccination intention using the PMT in the Iranian population.

\section{Methods}

\section{1. study design}

We conducted a cross-sectional study in the Iranian adult population 18 years and older and surveyed study participants from the first to the 30th of June 2020 with a web-based self-administered questionnaire. We made a questionnaire based on the conceptual framework of the PMT on the Porsline, an online survey platform in Iran. The questionnaire began with an information letter about the study's purpose, how to answer questions, and informed consent to participate in the study.

\subsection{Materials}

We asked participants about their demographic characteristics, including age, gender, education, and marital status. Also, we asked the participants about the perceived severity of COVID-19, perceived 
susceptibility to COVID-19, perceived self-efficacy in performing the COVID-19 vaccination and perceived response efficacy of COVID-19 vaccine, and intention to be vaccinated against COVID-19 whenever the vaccine was available. All answers were on 5-point Likert scales. The ethics committee of Zahedan University of Medical Sciences approved this study's protocol (IR.ZAUMS.REC.1399.015).

\subsection{Data analysis}

The analytical procedure consisted of two major tests: first, we performed confirmatory factor analysis (CFA), and also overall sample to check the goodness of fit of the hypothetical measurement model of each domain, postulated by protection motivation theory developers. We performed structural equation modeling (SEM) to test for the proposed model in the next step. For investigating the fit of each model, we calculated the chi-square $(\mathrm{X} 2)$ statistic. However, this well-known statistic is not a useful model fit index practically because of the detection of even trivial differences under a large sample size (18). Therefore, for more reliable results besides this test, we considered other goodness of fit indices like Comparative Fit Index (CFI), Tucker-Lewis Index (TLI), and Root Mean Square Error of Approximation (RMSEA) for a final decision about accepting or rejecting the hypothesis. A value of $C F I \geq 0.90, T L I \geq 0.90$, and RMSEA $\leq 0.08$ can support a good model fit (19). We chose full information maximum likelihood estimation as estimators. CFA and SEM run by Mplus 8.3 (20).

\section{Results}

\subsection{Participant Characteristics}

The average age of participants was $37.73 \pm 12.27$ years; $46.2 \%$ of them were male. $83.7 \%$ of participants had a university degree, $47.3 \%$ had an undergraduate degree, and $36.4 \%$ had a graduate degree. We reported the descriptive statistics of measured variables in the model in table 1, including skewness and kurtosis, which are indicators for univariate normality. The mean score range of items ranges from 3.208 to 4.475 , and standard deviation scores range from 0.723 to 1.164 . All items' skewness and kurtosis scores fall in the acceptable ranges of normality suggested by Kline (skewness does not exceed $|3|$ and kurtosis does not exceed |10|) (21). 


\begin{tabular}{|lllll}
\hline $\begin{array}{l}\text { Table1. Descriptive statistics of the items in the } \\
\text { measure }\end{array}$ & & & & \\
Construct and item & Mean & $\begin{array}{l}\text { Standard } \\
\text { deviation }\end{array}$ & Skewness & Kurtosis \\
\hline Perceived susceptibility & 17.478 & 1.164 & -0.07 & -0.278 \\
\hline SUS1 & 3.671 & 1.047 & -0.699 & -.064 \\
\hline SUS2 & 3.208 & 1.102 & 0.008 & -0.777 \\
\hline SUS3 & 3.295 & 1.101 & -0.125 & -0.791 \\
\hline SUS4 & 3.292 & 1.164 & -0.251 & -0.867 \\
\hline SUS5 & 4.004 & 0.870 & -0.915 & 0.943 \\
\hline Perceived severity & 22.045 & 3.841 & -1.957 & 5.088 \\
\hline SEV1 & 4.449 & 0.891 & -2.144 & 4.962 \\
\hline SEV2 & 4.417 & 0.775 & -1.863 & 4.982 \\
\hline SEV3 & 4.346 & 0.859 & -1.568 & 2.437 \\
\hline SEV4 & 4.458 & 0.723 & -1.738 & 4.454 \\
\hline SEV5 & 4.475 & 0.805 & -2.002 & 4.655 \\
\hline Perceived self-efficacy & 3.984 & 0.941 & -0.9 & 0.783 \\
\hline Perceived response efficacy & 8.220 & 3.751 & -0.981 & 1.937 \\
\hline R.E1 & 4.064 & 0.827 & -0.852 & 1.296 \\
\hline R.E2 & 4.155 & 0.833 & -1.212 & 2.194 \\
\hline Intention & 4.068 & 0.975 & -1.161 & 1.457 \\
\hline
\end{tabular}

We reported the Cronbach's alphas, the composite reliability (CR), and the average variance extracted (AVE) in table2. All Cronbach's alphas, CR and AVE, were greater than 0.70 , indicating good reliability and validity of items within a construct (table2).

\begin{tabular}{|c|c|c|c|}
\hline Table2. Reliability analysis & & & \\
\hline Cronbach's alphas & Composite reliability & Average variance extracted & \\
\hline Perceived susceptibility & 0.926 & 0.989 & 0.95 \\
\hline Perceived severity & 0.772 & 0.986 & 0.944 \\
\hline Perceived response efficacy & 0.848 & 0.969 & 0.941 \\
\hline
\end{tabular}

\subsection{Predictors of COVID-19 vaccination intention}


As mentioned earlier, the first step in testing SEM is to check whether the overall sample data fit the measurement model or not. The CFA analysis for all domains showed approximately acceptable CFI, TLI, and RMSEA values. Perceived susceptibility, perceived severity, perceived self-efficacy, and perceived response efficacy were predictors of intention in model 1 . As shown in table3, the goodness of fit incidence of the model was 655.911, P-value $<0.001, \mathrm{CFI}=0.960, \mathrm{TLI}=0.950$, and RMSEA $=0.081$. Although all goodness of fit indices were acceptable, perceived susceptibility was not significant, so we omitted perceived susceptibility to find a better model. Figure 1 also shows the graphical description of SEM analysis results. In this figure, you can see all coefficients for the measurement model and path analysis.

Table3. The goodness of fit index of models

\begin{tabular}{|lllllll} 
& $\begin{array}{l}\text { Chi- } \\
\text { square }\end{array}$ & df & $\begin{array}{l}\text { P- } \\
\text { value }\end{array}$ & RMSEA & CFI & TLI \\
\hline Measurement Model & 160.062 & 51 & $<0.001$ & 0.091 & 0.941 & 0.923 \\
\hline Model 1 & 184.937 & 69 & $<0.001$ & 0.081 & 0.948 & 0.932 \\
\hline Model 2 & 77.343 & 23 & $<0.001$ & 0.096 & 0.966 & 0.947 \\
\hline
\end{tabular}

In model 2, perceived severity, perceived self-efficacy, and perceived response efficacy were predictors of intention. As shown in table3 goodness of fit incidence of the model were 109.164, $\mathrm{P}$-value $<0.001, \mathrm{CFI}=$ $0.952, \mathrm{TLI}=0.933$, and RMSEA $=0.096$. In this model, all goodness of fit indices are acceptable, and this model can explain $61.5 \%$ of the variance of intention. Figure 2 also shows the graphical description of the results of the SEM analysis. In this figure, you can see all coefficients for the measurement model and path analysis. As shown in this figure, perceived severity to COVID-19 $(\beta=.17, p<.001)$, perceived selfefficacy about receiving the COVID-19 vaccine $(\beta=.26, p<.001)$, and the perceived response efficacy of the COVID-19 vaccine $(\beta=.70, p<.001)$ were significant predictors of vaccination intention. Response efficacy was the strongest predictor of COVID-19 vaccination intention.

\section{Discussion}

Identification of factors influencing the acceptance of the COVID-19 vaccine should begin before a vaccine becomes available. The current study applies the PMT to identify predictors of COVID-19 vaccination intention in the Iranian adult population. We used SEM to investigate the interrelationship between COVID-19 vaccination intention and perceived susceptibility, perceived severity, perceived selfefficacy, and perceived response efficacy. The results showed that if the COVID-19 vaccine is available, the PMT could be a good predictor for vaccination intention. Previous studies that have used the PMT to predict vaccination intention have shown its effectiveness $(22,23)$. A study that examined the predictor of seasonal influenza vaccination intention based on the PMT showed that the PMT accounted for $62 \%$ of vaccination intention variance (17). 
The current study showed that perceived susceptibility to COVID-19 was not a significant predictor of vaccination intention. Participants in this study received less than $70 \%$ of the total score of the perceived susceptibility construct, and this finding indicates that participants did not consider themselves high susceptible to COVID-19 infection. In studies examining the intention to vaccinate against H1N1 influenza, perceived susceptibility to influenza H1N1 virus did not predict vaccination intention $(24,25)$. Therefore, interventions should be designed and implemented by the health system to sensitize people to COVID-19. SEM showed that perceived severity to COVID-19, perceived self-efficacy about receiving the COVID-19 vaccine, and the perceived efficacy of the COVID-19 vaccine were significant predictors of vaccination intention. The three-factor model accounted for $61.5 \%$ of the total variance.

There is evidence that higher consideration of vaccination future consequences is associated with the perceived severity of the disease, greater perceived self-efficacy, and higher perceived effectiveness of the vaccine $(26,27)$. An extensive survey that examined the willingness to vaccinate against seven vaccinepreventable diseases in the United States showed that different degrees of risk are associated with the number of people willing to be vaccinated (28).

Additionally, a study examining the acceptability of the COVID-19 vaccine found that participants who reported higher levels of perceived severity of COVID-19 infection and perceived effectiveness of COVID19 vaccine were more likely to be willing to get vaccinated (5). This study indicates that the perceived response efficacy is the strongest predictor of COVID-19 vaccination intention among the PMT construct. Regarding the effectiveness of the COVID-19 vaccine, other studies revealed that belief in vaccine efficacy was significantly the probability of COVID-19 vaccine acceptance $(29,30)$.

However, there is evidence that other factors can play a decisive role in influenza vaccination, despite understanding its effectiveness (31). The previous research shows that perceived self-efficacy is one of the most critical factors in adherence to COVID-19 preventive measures (32). Perceived self-efficacy refers to a sense of control over novel or difficult situations and challenges through decent behavior (33). In behaviors such as vaccination that do not involve long-term treatment adherence, self-efficacy is a determinant of intention and behavior (34). In a previous study that used PMT to predict staying at home during the COVID-19 pandemic in the Japanese population, self-efficacy was a predictor.

Like this study's results, perceived severity leads to threat appraisal more than perceived vulnerability, and perceived self-efficacy and perceived response efficiency leads to coping appraisal (35). Therefore, to encourage people to get vaccinated against COVID-19, more emphasis should be placed on perceived severity and perceived response efficiency. Because vaccination intention and actual vaccination uptake are related (36), identifying factors influencing vaccination intention before the availability of the COVID19 vaccine can pave the way for community acceptance of the vaccine. Therefore, future intervention to increase COVID-19 vaccine acceptance can consider the PMT as a conceptual framework.

Readers should interpret our findings in light of the following study limitations. First, the COVID-19 vaccine is not yet available, and individuals' answers to questions about vaccine efficacy and selfefficacy related to the vaccine may differ when the vaccine is available. Also, the distribution and cost of 
the vaccine are not known. If a vaccine provides in the future, the people who have access to the vaccine may have different characteristics from the participants in this study. Second, because we selected participants to study through an online survey platform, the findings may be prone to selection bias. Third, this study's data were self-reported, and participants' responses may be social desirability biased.

\section{Conclusions}

The current study identified factors associated with the COVID-19 vaccination intention. Understanding the factors influencing vaccination can help health policymakers increase vaccine acceptance. Programs designed to increase the vaccination rate after the availability of the COVID-19 vaccine can include interventions on the severity of the COVID-19, the self-efficacy of individuals receiving the vaccine, and the effectiveness of the vaccine in preventing infection.

\section{Declarations}

\section{Ethics approval and consent to participate}

The ethics committee of Zahedan University of Medical Sciences approved this study's protocol (IR.ZAUMS.REC.1399.015). Participants expressed informed consent to participate in the study before beginning to respond to the online questionnaire.

\section{Consent for publication}

Not applicable.

\section{Availability of data and materials}

The datasets used and analyzed during the current study are available from the corresponding author on reasonable request.

\section{Competing interests}

The authors declare that they have no competing interests.

\section{Funding}

The Zahedan University of Medical Sciences supported the study.

\section{Authors' contributions}

HOA and MS participated in designing the study; ZS and MM participated in data analysis; HOA and AAM interpreted the results and prepared the manuscript. All authors read and approved the final manuscript.

\section{Acknowledgments}


The authors would like to thank all the participants in this study.

\section{Abbreviation}

PMT $=$ Protection Motivation Theory, SEM = Structural Equation Modeling, CFA = Confirmatory Factor Analysis, CFI = Comparative Fit Index, TLI = Tucker-Lewis Index, RMSEA = Root Mean Square Error of Approximation, $\mathrm{CR}=$ Composite Reliability $(\mathrm{CR}), \mathrm{AVE}=$ Average Variance Extracted

\section{References}

1. Orenstein WA, Ahmed R. Simply put: vaccination saves lives. National Acad Sciences; 2017. https://doi.org/10.1073/pnas.1704507114

2. World Health Organization. Assessment Report of the Global Vaccine Action Plan. Strategic Advisory Group of Experts on Immunization. Geneva: WHO. 2017.

3. Yang P, Wang X. COVID-19: a new challenge for human beings. Cellular \& molecular immunology. 2020;17(5):555-7. https://doi.org/10.1038/s41423-020-0407-x

4. World Health Organization. DRAFT landscape of COVID-19 candidate vaccines. World. 2020.

5. Reiter PL, Pennell ML, Katz ML. Acceptability of a COVID-19 vaccine among adults in the United States: How many people would get vaccinated? Vaccine. 2020;38(42):6500-7. https://doi.org/10.1016/j.vaccine.2020.08.043

6. Control CfD, Prevention. Flu vaccination coverage, United States, 2018-19 influenza season. 2019.

7. Sheldenkar A, Lim F, Yung CF, Lwin MO. Acceptance and uptake of influenza vaccines in Asia: a systematic review. Vaccine. 2019;37(35):4896-905. https://doi.org/10.1016/j.vaccine.2019.07.011

8. Tanjani PT, Babanejad M, Najafı F. Influenza vaccination uptake and its socioeconomic determinants in the older adult Iranian population: A national study. American Journal of Infection Control. 2015;43(5):e1-e5. https://doi.org/10.1016/j.ajic.2015.02.001

9. Larson HJ, De Figueiredo A, Xiahong Z, Schulz WS, Verger P, Johnston IG, et al. The state of vaccine confidence 2016: global insights through a 67-country survey. EBioMedicine. 2016;12:295-301. https://doi.org/10.1016/j.ebiom.2016.08.042

10. Zaraket $\mathrm{H}$, Melhem N, Malik M, Khan WM, Dbaibo G, Abubakar A. Review of seasonal influenza vaccination in the Eastern Mediterranean Region: Policies, use and barriers. Journal of infection and public health. 2020. https://doi.org/10.1016/j.jiph.2020.02.029

11. Lane S, MacDonald NE, Marti M, Dumolard L. Vaccine hesitancy around the globe: Analysis of three years of WHO/UNICEF Joint Reporting Form data-2015-2017. Vaccine. 2018;36(26):3861-7. https://doi.org/10.1016/j.vaccine.2018.03.063

12. Neumann-Böhme S, Varghese NE, Sabat I, Barros PP, Brouwer W, van Exel J, et al. Once we have it, will we use it? A European survey on willingness to be vaccinated against COVID-19. Springer; 2020. https://doi.org/10.1007/s10198-020-01208-6 
13. Dubé E, MacDonald NE. How can a global pandemic affect vaccine hesitancy? : Taylor \& Francis; 2020. https://doi.org/10.1080/14760584.2020.1825944

14. Betsch C, Böhm R, Chapman GB. Using behavioral insights to increase vaccination policy effectiveness. Policy Insights from the Behavioral and Brain Sciences. 2015;2(1):61-73. https://doi.org/10.1177/2372732215600716

15. Tannenbaum MB, Hepler J, Zimmerman RS, Saul L, Jacobs S, Wilson K, et al. Appealing to fear: A meta-analysis of fear appeal effectiveness and theories. Psychological bulletin. 2015;141(6):1178. https://doi.org/10.1037/a0039729

16. Ruiter RA, Kessels LT, Peters GJY, Kok G. Sixty years of fear appeal research: Current state of the evidence. International journal of psychology. 2014;49(2):63-70. https://doi.org/10.1002/ijop.12042

17. Ling $M$, Kothe EJ, Mullan BA. Predicting intention to receive a seasonal influenza vaccination using Protection Motivation Theory. Social Science \& Medicine. 2019;233:87-92. https://doi.org/10.1016/j.socscimed.2019.06.002

18. Cheung GW, Rensvold RB. Evaluating Goodness-of-Fit Indexes for Testing Measurement Invariance. Structural Equation Modeling: A Multidisciplinary Journal. 2002;9(2):233-55. https://doi.org/10.1207/s15328007sem0902_5

19. Hu L-t, Bentler PM. Cutoff criteria for fit indexes in covariance structure analysis: Conventional criteria versus new alternatives. Structural Equation Modeling. 1999;6(1):1-55. https://doi.org/10.1080/10705519909540118

20. Muthén LK, Muthen B. Mplus user's guide: Statistical analysis with latent variables, user's guide: Muthén \& Muthén; 2017.

21. Kline RB. Principles and practice of structural equation modeling: Guilford publications; 2015.

22. Camerini A-L, Diviani N, Fadda M, Schulz PJ. Using protection motivation theory to predict intention to adhere to official MMR vaccination recommendations in Switzerland. SSM-Population Health. 2019;7:100321. https://doi.org/10.1016/j.ssmph.2018.11.005

23. Liu C, Nicholas S, Wang J. The association between protection motivation and hepatitis $b$ vaccination intention among migrant workers in Tianjin, China: a cross-sectional study. BMC Public Health. 2020;20(1):1-10. https://doi.org/10.1186/s12889-020-09292-2

24. Lau JT, Yeung NC, Choi K, Cheng MY, Tsui H, Griffiths S. Factors in association with acceptability of $\mathrm{A} / \mathrm{H} 1 \mathrm{~N} 1$ vaccination during the influenza $\mathrm{A} / \mathrm{H} 1 \mathrm{~N} 1$ pandemic phase in the Hong Kong general population. Vaccine. 2010;28(29):4632-7. https://doi.org/10.1016/j.vaccine.2010.04.076

25. Coe AB, Gatewood SB, Moczygemba LR. The use of the health belief model to assess predictors of intent to receive the novel (2009) H1N1 influenza vaccine. Innovations in pharmacy. 2012;3(2):1. https://doi.org/10.24926/iip.v3i2.257

26. Kim J, Kim Y. Consideration of future consequences and predictability: Examining six health behaviors with different levels of perceived severity. The Social Science Journal. 2020:1-9. https://doi.org/10.1080/03623319.2020.1808768 
27. Nan X, Kim J. Predicting H1N1 vaccine uptake and H1N1-related health beliefs: The role of individual difference in consideration of future consequences. Journal of health communication. 2014;19(3):376-88. https://doi.org/10.1080/10810730.2013.821552

28. Baumgaertner B, Ridenhour BJ, Justwan F, Carlisle JE, Miller CR. Risk of disease and willingness to vaccinate in the United States: A population-based survey. PLoS Medicine. 2020;17(10):e1003354. https://doi.org/10.1371/journal.pmed.1003354

29. Pogue K, Jensen JL, Stancil CK, Ferguson DG, Hughes SJ, Mello EJ, et al. Influences on Attitudes Regarding Potential COVID-19 Vaccination in the United States. Vaccines. 2020;8(4):582. https://doi.org/10.3390/vaccines8040582

30. Wang J, Jing R, Lai X, Zhang H, Lyu Y, Knoll MD, et al. Acceptance of COVID-19 Vaccination during the COVID-19 Pandemic in China. Vaccines. 2020;8(3):482. https://doi.org/10.3390/vaccines8030482

31. Lutz CS, Fink RV, Cloud AJ, Stevenson J, Kim D, Fiebelkorn AP. Factors associated with perceptions of influenza vaccine safety and effectiveness among adults, United States, 2017-2018. Vaccine. 2020;38(6):1393-401. https://doi.org/10.1016/j.vaccine.2019.12.004

32. Chong YY, Chien WT, Cheng HY, Chow KM, Kassianos AP, Karekla M, et al. The Role of Illness Perceptions, Coping, and Self-Efficacy on Adherence to Precautionary Measures for COVID-19. International Journal of Environmental Research and Public Health. 2020;17(18):6540. https://doi.org/10.3390/ijerph17186540

33. Warner LM, Schwarzer R. Self-Efficacy and Health. The Wiley Encyclopedia of Health Psychology. 2020:605-13. https://doi.org/10.1002/9781119057840.ch111

34. Fall E, Izaute M, Chakroun-Baggioni N. How can the health belief model and self-determination theory predict both influenza vaccination and vaccination intention? A longitudinal study among university students. Psychology \& health. 2018;33(6):746-64. https://doi.org/10.1080/08870446.2017.1401623

35. Okuhara T, Okada H, Kiuchi T, editors. Predictors of Staying at Home during the COVID-19 Pandemic and Social Lockdown based on Protection Motivation Theory: A Cross-Sectional Study in Japan. Healthcare; 2020: Multidisciplinary Digital Publishing Institute. https://doi.org/10.3390/healthcare8040475

36. Gargano LM, Painter JE, Sales JM, Morfaw C, Jones LM, Murray D, et al. Seasonal and 2009 H1N1 influenza vaccine uptake, predictors of vaccination, and self-reported barriers to vaccination among secondary school teachers and staff. Human Vaccines. 2011;7(1):89-95. https://doi.org/10.4161/hv.7.1.13460

\section{Figures}




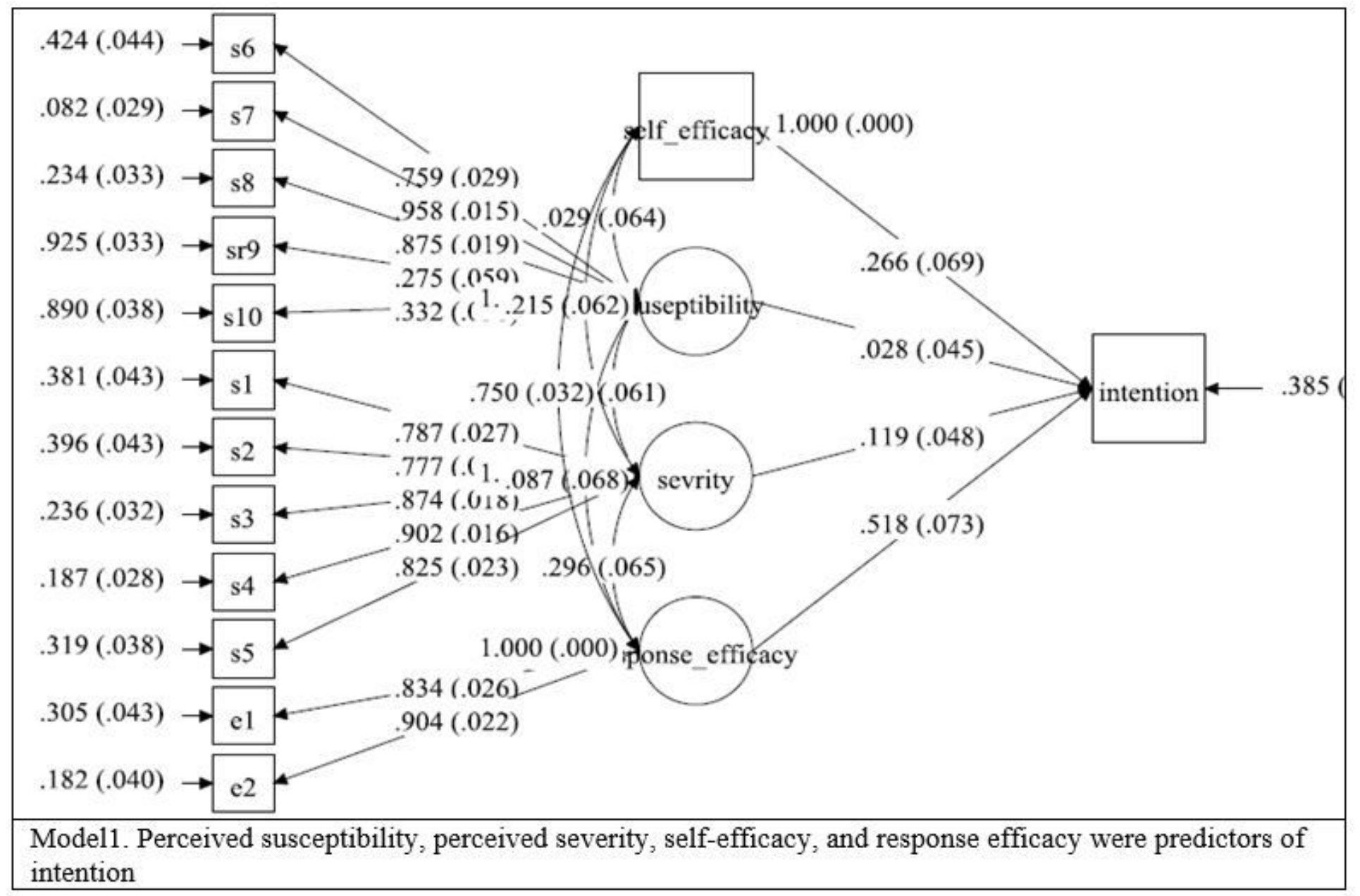

Figure 1

Perceived susceptibility, perceived severity, self-efficacy, and response efficacy were predictors of intention 


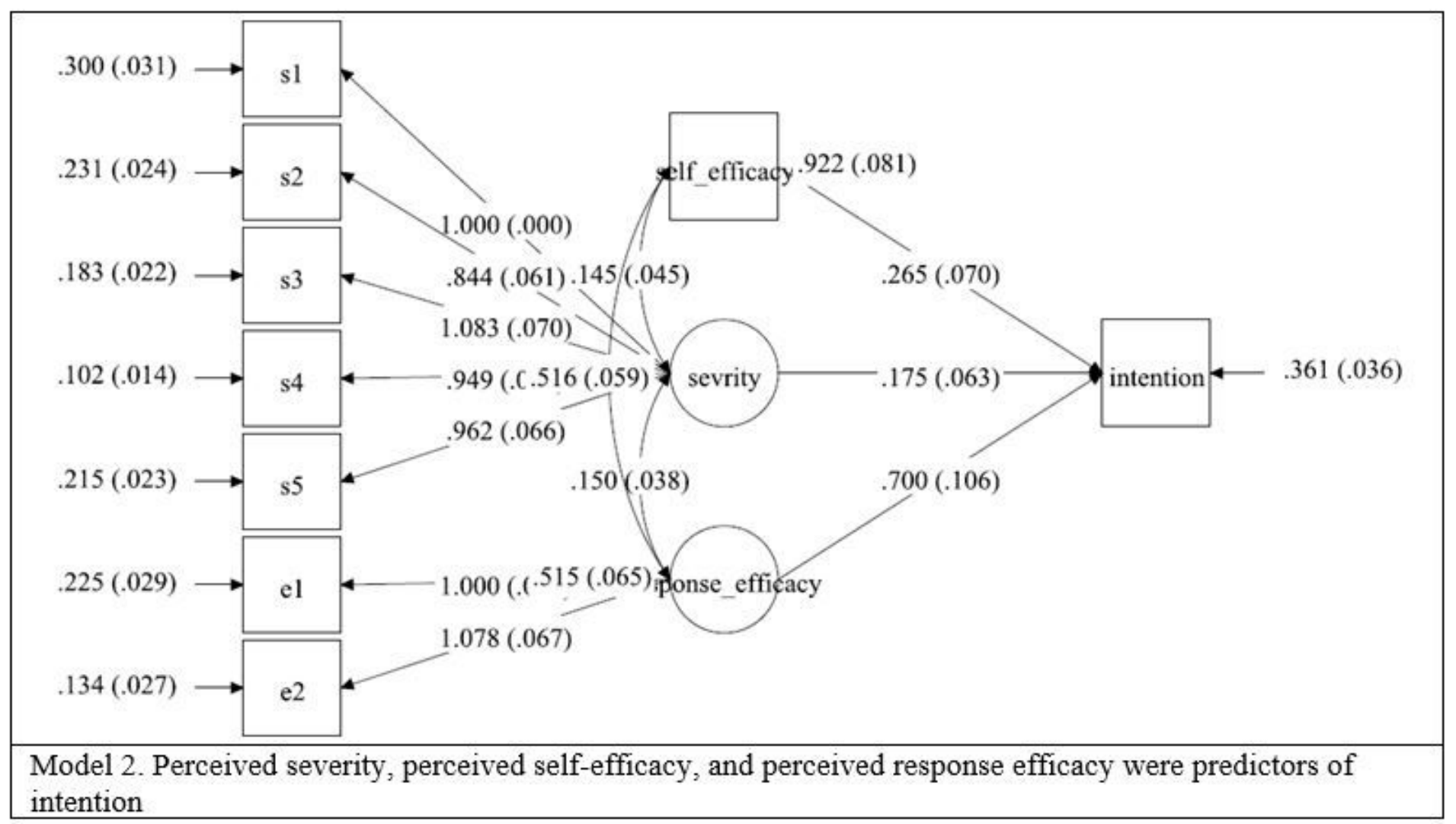

Figure 2

Perceived severity, perceived self-efficacy, and perceived response efficacy were predictors of intention 